\title{
Structural Study of CuNCN and Its Theoretical Implications: A Case of a Resonating-Valence-Bond State?
}

\author{
Andrei L. Tchougréeff, ${ }^{\dagger,}$ Xiaohui Liu, ${ }^{\dagger}$ Paul Müller, ${ }^{\dagger}$ Wouter van Beek, ${ }^{\S}$ Uwe Ruschewitz, ${ }^{\|}$ \\ and Richard Dronskowski* ${ }^{\dagger}$ \\ ${ }^{\dagger}$ Institute of Inorganic Chemistry, RWTH Aachen University, Landoltweg 1, D-52056 Aachen, Germany \\ ${ }^{\ddagger}$ Lomonosov Moscow State University, Department of Chemistry \& Poncelet Laboratory of Mathematics in Interaction with Physics \\ and Informatics, Independent University of Moscow, Moscow Center for Continuous Mathematical Education, Bolshoi Vlasevsky \\ Per. 11, 119002 Moscow, Russia \\ ${ }^{\S}$ Swiss-Norwegian Beamlines at ESRF, BP 220, Grenoble, 38043, France, and Dipartimento di Scienze e Tecnologie Avanzate and \\ NanoSistemi IC, Universita del Piemonte Orientale, Via Michel 11, Alessandria 15121, Italy \\ "Institute of Inorganic Chemistry, University of Cologne, Greinstrasse 6, D-50939 Köln, Germany
}

Supporting Information

\begin{abstract}
We present the results of a high-resolution low-temperature synchrotron structural study of the nitrogen-containing analogue of cupric oxide, copper carbodiimide $(\mathrm{CuNCN})$. The material clearly manifests a surprising nonmonotonous behavior of the $a$ lattice parameter as a function of the temperature: its decrease with decreasing temperature turns to a distinguishable increase below $100 \mathrm{~K}$. The onset temperature of this anomaly nearly coincides with that of the onset of the activation temperature dependence, as seen from the magnetic susceptibility, which has been tentatively attributed to the formation of a gapped 2D spin-liquid (resonating-valence-bond) state. In accord with this assumption, we develop a theory relating the temperature behavior of the spin susceptibility to that of the lattice parameter, which semiquantitatively agrees with the observed behavior.
\end{abstract}

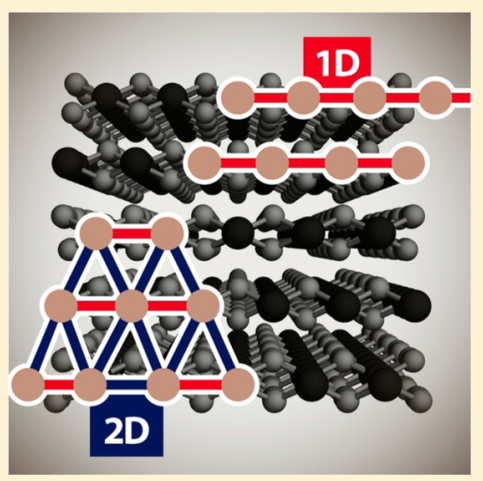

SECTION: Molecular Structure, Quantum Chemistry, and General Theory

$\mathrm{P}$ auling's ingenious idea of a (resonating) valence-bond state $^{1-3}$ is a chemical milestone in describing the electronic structure of the benzene molecule as a superposition (resonance) of its valence structures corresponding to different pairings of the $\pi$ electrons. Within solid-state physics, Anderson $^{4}$ suggested the resonating-valence-bond (RVB) state as a possible model for a new type of insulator, and this model turned out to be extremely helpful ${ }^{5}$ to explain the properties of the high-temperature cuprate superconductors. ${ }^{6}$ Since then, this hypothetical ground state is being sought in many materials, for example, those where one expects structurally stipulated frustration of the magnetic exchange interactions to induce RVB-type ground states. ${ }^{7}$ In particular, the "organic" conductors of the family $\kappa$-(BEDT$\mathrm{TTF})_{2} \mathrm{Cu}_{2}(\mathrm{CN})_{3}{ }^{8}$ and the halocuprates of the formula $\mathrm{Cs}_{2} \mathrm{CuCl}_{4}$ and $\mathrm{Cs}_{2} \mathrm{CuBr}_{4}{ }^{9-11}$ are considered to be highly probable RVB candidates. From a more theoretical perspective, the possibility of a $2 \mathrm{D}$ spin-liquid state has been recently confirmed based on large-scale quantum Monte Carlo simulations. ${ }^{12}$ Nonetheless, the characteristic temperatures of these complex phases are on the order of only a few kelvins as compared with hundreds of kelvins (e.g., the Néel temperatures) characteristic for the copper-oxide-based materials. The parent compound of all cuprates, cupric oxide $(\mathrm{CuO})$, behaves rather normally because its antiferromagnetic phase transitions apparently result from various 1D antiferromagnetic chain interactions. $^{13}$

Doping (or substitution) of the copper site looks rather straightforward to "tune" cupric oxide electronically, but an alternative approach consists of chemically modifying the anion such as in the nitrogen-containing $\mathrm{CuO}$ analogue called copper carbodiimide ( $\mathrm{CuNCN})$. In this compound, the $\mathrm{NCN}^{2-}$ carbodiimide anion (not the $\mathrm{N}^{3-}$ nitride anion) replaces the $\mathrm{O}^{2-}$ oxide anion for charge-balance reasons, in the spirit of a " $\mathrm{N}^{2-"}$ (divalent nitride) species that does not exist. CuNCN has only recently been synthesized in pure form, and its structure has been determined. ${ }^{14}$ In the orthorhombic crystal structure depicted in Figure 1, one finds corrugated layers of $\mathrm{Cu}^{2+}$ ions that experience a rather typical first-order JahnTeller distortion with four $\mathrm{Cu}-\mathrm{N}$ distances around $2.0 \AA$ and two more at $\sim 2.6 \AA$.

$\mathrm{CuNCN}$ is a most peculiar material. The first magnetic susceptibility data showed a very small and almost temperature-

Received: October 24, 2012

Accepted: October 31, 2012 


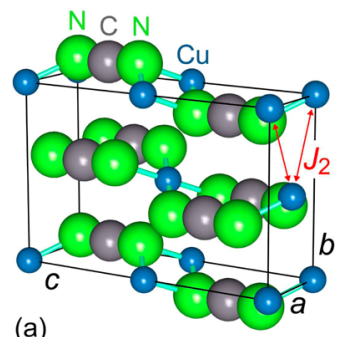

(a)

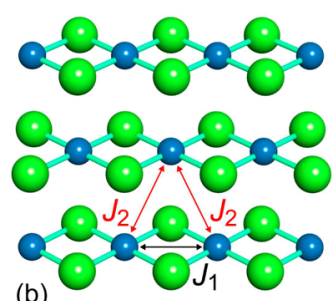

Figure 1. (a) Crystal structure of $\mathrm{CuNCN}$ and (b) the dominating exchange parameters existing in its $a b$ plane. A stronger $J_{1}$ extends in the $a$ direction, whereas a weaker $J_{2}$ extends along the $b \pm a$ directions.

independent signal, and GGA $(+U)$ electronic-structure calculations were in accord with antiferromagnetic spin exchange paths within the $a b$ plane, compatible with a $2 \mathrm{D} S=1 / 2$ frustrated triangular Heisenberg quantum antiferromagnet. ${ }^{15}$ Puzzlingly enough, it was impossible to observe magnetic Bragg peaks for perfectly crystalline $\mathrm{CuNCN}$ at very low temperature $(4.6 \mathrm{~K})$, even when operating with highly polarized neutrons ${ }^{16}$ such that coherent magnetic scattering indicative of ordering of unpaired electrons could clearly be excluded. This observation makes whatever antiferromagnetic electronic-structure models ${ }^{17}$ proposed in the sequel extremely unlikely.

More recent physical measurements, that is, magnetic susceptibility, electron-spin resonance, nuclear magnetic resonance, and finally muon-spin relaxation studies, also reveal that classical magnetic ordering is absent down to the lowest temperatures in the millikelvin range. ${ }^{18}$ Instead, one observes a fairly temperature-independent paramagnetism down to ca. 100 $\mathrm{K}$, which changes to a considerable decrease in the susceptibility evolving in an activation-like fashion. Incidentally, there is no susceptibility difference between the field-cooled and zero-field-cooled samples, which makes the spin-glass picture questionable as well. All that did not receive unequivocal interpretation so far. Either an unexpected inhomogeneous magnetic ground state or a gapped dynamical spin-liquid state has been proposed to install below $100 \mathrm{~K}$, both to be attributed to a peculiar fragility of the electronic structure against weak perturbations due to geometrical frustration. This immediately asks for a high-resolution structural analysis in that alternative (RVB?) ground states might manifest themselves in the structural parameters, even if the structural changes were extremely small, simply because the latter would then occur by the reconstruction of only a small fraction of all electrons. This study aims to bring new insight into the crystal structure of $\mathrm{CuNCN}$ at low temperatures $(5 \mathrm{~K})$ with very high resolution and accuracy. On the basis of the observed features, we provide a tentative theoretical explanation in the sense of the longsought resonating valence-bond state.

To start with, the unit cell volume deduced from $T$ dependent synchrotron data decreases from room to very low temperature (Supporting Information), just as expected for regular solids. For the course of the individual lattice parameters, depicted in Figure 2, the behavior is more complex. Whereas the $b$ lattice parameter looks rather unobtrusive, the behavior of the $a$ and $c$ lattice parameters turns to be more chaotic at $\sim 150 \mathrm{~K}$ and, at least for the a parameter, it clearly inverts (!) below $100 \mathrm{~K}$ : there is a distinct increase in $a$ while the temperature decreases. Incidentally, this increase sets in at approximately the same temperature where the ESR and SQUID measured magnetic susceptibilities begin to decrease. ${ }^{18}$

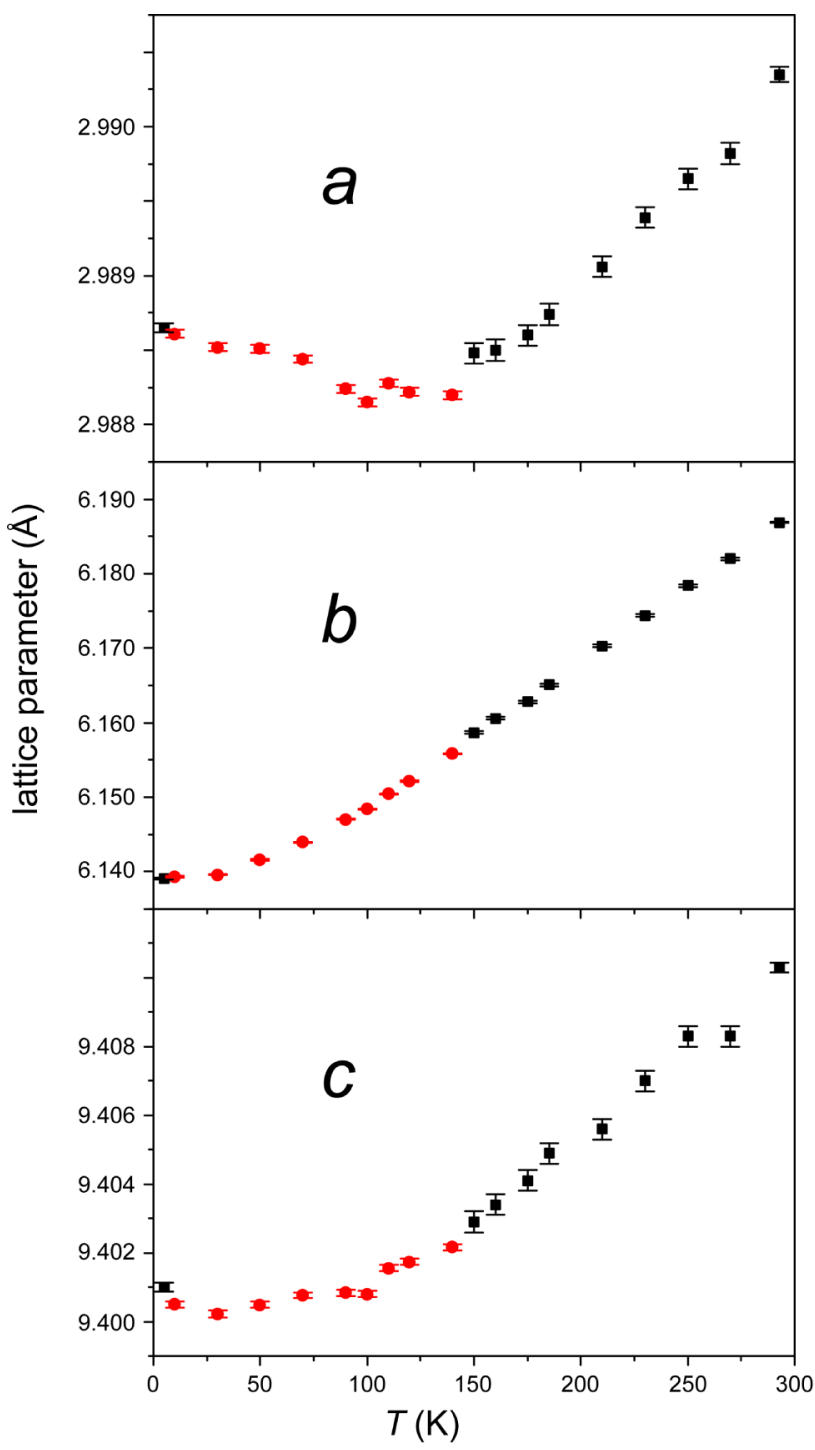

Figure 2. Course of the lattice parameters $a, b$, and $c$ of $\mathrm{CuNCN}$ as a function of the temperature. Data of the first run $(\lambda=0.50195 \AA)$ are given in black, and those of the second run $(\lambda=0.50350 \AA)$ are given in red. More details are given in the Experimental Methods section.

It has been conjectured ${ }^{19}$ that the switch between the temperature-independent paramagnetism above 80-100 K and the magnetic susceptibility vanishing according to an activation law below this temperature may be attributed to a transition between the two magnetically disordered spin-liquid phases on the anisotropic triangular lattice: a high-temperature gapless (1D resonating valence bond: 1D-RVB) phase and a low-temperature gapped phase (2D resonating valence bond: 2D-RVB). ${ }^{20-26}$ Both scenarios have been sketched in Figure 3. In the sequel, we will show that the structural change observed below $100 \mathrm{~K}$ may indeed be explained using the same hypothesis of the two spin-liquids.

To understand the phenomenon, we recall that the ESR experiments ${ }^{18}$ unequivocally attribute the magnetic response of $\mathrm{CuNCN}$ to that of the spins- $1 / 2$ residing on the $\mathrm{Cu}$ sites to account for the observed characteristic $g$ factors. The interactions between these spins presumably correspond to the Heisenberg Hamiltonian

$$
\Sigma_{\mathbf{r}, \tau} J_{\tau} S_{\mathbf{r}} \boldsymbol{S}_{\mathbf{r}+\tau}
$$




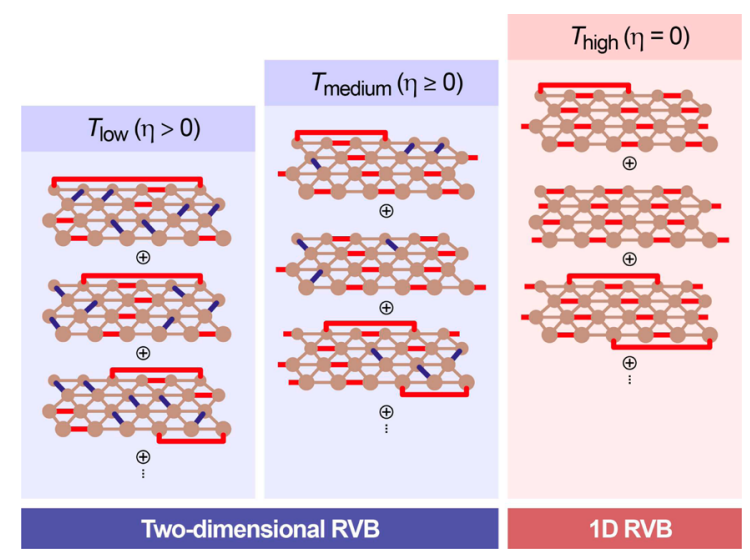

Figure 3. Highly schematic representation of the two resonatingvalence-bond (RVB) states within CuNCN. At high $T$ (right), a strictly 1D-RVB state persists as a superposition of a large number of microstates with horizontally (along the $a$ direction) spin-paired $\mathrm{Cu}-$ $\mathrm{Cu}$ couples, where each bond is a singlet, that is, a paired valence-bond state of two electrons: $1 / \sqrt{2}\left(\boldsymbol{c}_{\mathbf{r}_{\uparrow} \uparrow}^{+} \boldsymbol{c}_{\mathbf{r}^{\prime} \downarrow}^{+}+\boldsymbol{c}_{{ }_{\mathbf{r} \downarrow} \downarrow}^{+} c_{\mathbf{r}^{\prime} \uparrow}^{+}\right)$. At medium $T$ (lower than $100 \mathrm{~K}$, middle), a 2D-RVB state is composed of microstates including both horizontal $(a)$ and also oblique $(a \pm b) \mathrm{Cu}-\mathrm{Cu}$ couplings in the triangular net. At the lowest temperatures (left), the depletion of the order parameter $\boldsymbol{\xi}$ (proportional to the bond order) in the 2D-RVB state is equivalent to a lowering fraction of microstates with horizontal $\mathrm{Cu}-\mathrm{Cu}$ couplings, thus mirrored by a slightly enlarged $\mathrm{Cu}-\mathrm{Cu}$ distance.

where $J_{\tau}$ takes one of the two values $J_{1}$ and $J_{2}$, as depicted in Figure 1, and each spin has six nearest neighbors whose interactions are taken into account. $S_{\mathrm{r}}$ is the spin operator on site $\mathbf{r}$, and $\tau$ refers to one of the nearest-neighbor vectors. (See the Supporting Information.)

Because of the frustration occurring in the triangular lattice and depending on the ratio of the exchange parameters $J_{1}$ and $J_{2}$, the electronic spins arrange in a 1D-RVB or a $2 \mathrm{D}-\mathrm{RVB}$ state. As shown in Figure 3, the 1D-RVB state is a superposition of microstates with spin pairings between $\mathrm{Cu}^{2+}$ that extend exclusively within the chains lying along the crystallographic $a$ direction. This state presumably exists at higher temperature, whereas at medium temperature ( $<100 \mathrm{~K}$, middle of Figure 3), a $2 \mathrm{D} \mathrm{RVB}$ state sets in. For the 2D-RVB state, spin pairings may also extend obliquely to the $a$ direction so that microstates with pairings between all possible nodes of the triangular lattice enter the total state. Nonetheless, forming a microstate with an oblique bond requires that at least two longitudinal bonds are broken because each $\mathrm{Cu}^{2+}$ ion may take part in only one pairing within each microstate. Thus, the 2D-RVB state is composed of microstates including both $a$-longitudinal and also oblique $\mathrm{Cu}-$ $\mathrm{Cu}$ couplings in the triangular net. At lowest temperatures (left of Figure 3), the number of oblique bonds increases, thus lowering the fraction of the $a$-longitudinal bonds (drastically exaggerated in Figure 3 ). The depletion of the order parameter $\boldsymbol{\xi}$ (being proportional to the bond order, see Supporting Information) in the 2D-RVB state is equivalent to a lowering fraction of microstates with $a$-longitudinal $\mathrm{Cu}-\mathrm{Cu}$ couplings.

In the mean-field picture of the RVB state, ${ }^{27}$ as adjusted for anisotropic triangular lattices, ${ }^{19,21,22,28}$ the amplitudes of the microstates with different pairings depicted in Figure 3 entering the macroscopic 1D-RVB and 2D-RVB states are integrally characterized either by one or two nonvanishing order parameters $\boldsymbol{\xi}$ and $\boldsymbol{\eta}$ (see Supporting Information). The relation between the two in the gapped low-temperature 2D-RVB state, where both order parameters depart from zero, has been obtained $^{28}$ as (Supporting Information, eq 6):

$$
\boldsymbol{\xi}=1 / \pi \sqrt{2}-\pi \sqrt{2} \boldsymbol{J}_{2} \boldsymbol{\eta}^{2} / \boldsymbol{J}_{1}
$$

This is in remarkable agreement with a previous numerical result. $^{22}$ There it was shown that in the 2D-RVB state onset region $(\boldsymbol{\eta} \neq 0)$ the $\boldsymbol{\xi}$ parameter manifests a very weak depletion as compared with its $1 \mathrm{D}-\mathrm{RVB}(\boldsymbol{\eta}=0)$ value. Because $\boldsymbol{\xi}$ is by definition proportional to the bond order between the neighboring atoms along the $a$ direction (Supporting Information), this depletion directly relates to the observed structural change, as has been reasoned ${ }^{28}$ in line with a general theoretical framework. ${ }^{29-31}$ A similar construct has been used in the cuprate RVB context. ${ }^{32}$

Let us first assume that the lattice contribution to the total energy per copper site can be harmonically approximated as a function of the $\mathrm{Cu}-\mathrm{Cu}$ separation along $a$. In fact, the $\mathrm{Cu}-\mathrm{Cu}$ separation is identical to the $a$ lattice parameter. This yields the term $K\left(a-a_{0}\right)^{2} / 2$ in the energy. Here $K$ is an effective elastic "spring" constant and $a_{0}$ is an equilibrium lattice parameter under the assumption that the spin contribution to the energy has been turned off. We then express the various energy contributions in terms of $\xi$, which yields $-6 \sqrt{2} J_{1} \xi / \pi$ for the "kinetic" energy (see Supporting Information and ref 28) and $6 J_{1} \xi^{2}$ for the potential energy. By making use of the virial theorem, we get $-6 J_{1} \xi^{2}$ for the spin contribution to the total energy. If we further assume a linear dependence of the effective exchange integral on the interatomic separation, as given by $J_{1}=J_{1}\left(a_{0}\right)+J^{\prime}{ }_{1}\left(a-a_{0}\right)$, then we easily arrive to the analogue of the famous bond-length versus bond-order relationship: ${ }^{33}$

$$
a=a_{0}+6 \sqrt{2} \boldsymbol{J}_{1}^{\prime} \xi / \pi K
$$

Here $a_{0}$ is the equilibrium lattice parameter while the spins do contribute to the energy. Obviously, the lattice parameter is going to increase, as observed, provided the effective exchange integral decreases with an increasing interatomic separation $\left(\boldsymbol{J}^{\prime}{ }_{1}\right.$ $<0)$. On this basis, we may state that a decrease in $\boldsymbol{\xi}$ will increase the equilibrium interatomic separation in the $a$ direction, and this is seen from the experiment (Figure 2). Finally, we reiterate the tentative interpretation ${ }^{18}$ of the ESRmeasured magnetic susceptibility in the form of a gradual development of the energy gap in the spectrum of fermionic quasiparticles below ca. $100 \mathrm{~K}$. In that study, the gap has been tentatively identified with the gap of $3 \sqrt{ } 2 J_{2} \eta$ characteristic for the 2D-RVB gapped spin liquid. The ESR susceptibility measurements interpreted in that sense provide an estimate for the temperature dependence of the $\boldsymbol{\eta}$ order parameter. Combining eqs 2 and 3, we arrive at the course of the equilibrium interatomic separation in the $2 \mathrm{D}-\mathrm{RVB}$ state

$$
\delta a=\Lambda \eta^{2} ; \quad \Lambda=-6 J_{2} J_{1}^{\prime} / J_{1} K
$$

which immediately shows that the $a$ lattice parameter in CuNCN must manifest the same temperature trend as the $2 \mathrm{D}$ RVB gap $\left(J^{\prime}{ }_{1}<0\right)$, although with somewhat damped amplitude due to its second order in a small quantity $\boldsymbol{\eta}$. The theory ${ }^{28}$ allows us to establish a semiquantitative correspondence between the ESR-measured energy gap and the observed structural variation. As mentioned before, the gap equals $3 \sqrt{ } 2 J_{2} \boldsymbol{\eta}$, which together with the value of $J_{2}=540 \mathrm{~K}$ allowed estimation of the temperature dependence of $\boldsymbol{\eta}$ in ref 18. By taking these $\boldsymbol{\eta}$ values, we have tried to model the temperature 
dependence of the $a$ lattice variation using eq 4 . The result is presented in Figure 4. Indeed, the lattice-parameter variation is

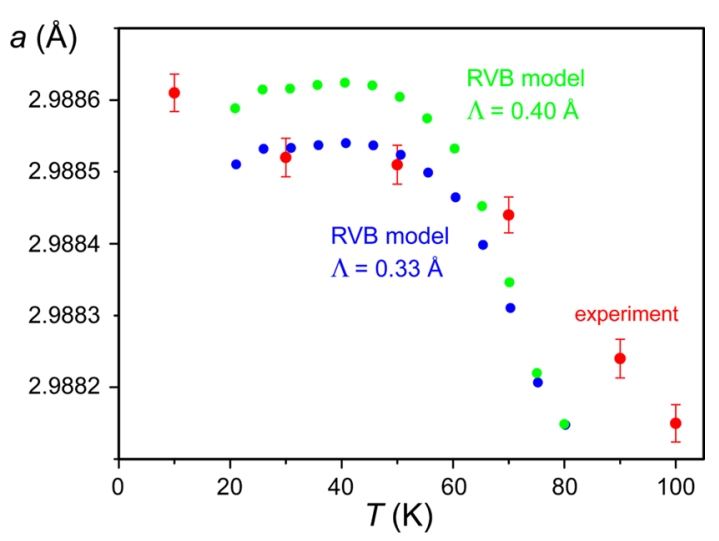

Figure 4. Course of the variation of the $a$ lattice parameter of $\mathrm{CuNCN}$ as a function of the temperature, with the experimental lowtemperature data $(\lambda=0.50350 \AA)$ and their standard deviations given in red. The blue and green model values have been calculated from the ESR-based data ${ }^{18}$ on the magnitude of the order parameter $\boldsymbol{\eta}$. The standard deviations in $T$ are smaller than $0.2 \mathrm{~K}$.

in qualitative agreement with the experimental data from our synchrotron diffraction study. A semiquantitative agreement can be achieved with coefficients $\Lambda$ of 0.33 and $0.4 \AA$. Using these values and the estimate ${ }^{18}$ for $J_{1}=2300 \mathrm{~K}$, one further calculates, respectively, 0.23 and $0.28 \AA$ for the effective spinphonon coupling constant $J^{\prime}{ }_{1} / K$, which seem to be acceptable values as well.

We also note that even within the achieved accuracy there is not the slightest trace of any superstructure formation in either crystallographic direction, thereby excluding the spin-Peierls scenario $^{34}$ for $\mathrm{CuNCN}$ (see also below), equivalent to the bond-order wave state. ${ }^{35,36}$ For the adopted triangular lattice model in the $a b$ plane, this fact allows us to make one more estimate for the relative strength of the interactions involved: the characteristic spin-phonon energy $\left(J^{\prime}{ }_{1}\right)^{2} / K$ must be smaller than the oblique exchange $J_{2}$, both taking part of the effective interaction in corresponding mean-field theories. More characteristic is the fact that the spin-Peierls state does not develop in the $c$ direction either, where it could be a good ground-state candidate within the picture of a strong exchange extended in this direction and mediated by the $\mathrm{NCN}^{2-}$ units as actively promulgated in ref 17 . Had the spin-Peierls been there it would explain at least two key features of $\mathrm{CuNCN}$ : the activation dependence of susceptibility due to opening of the spin-Peierls gap and the absence of the magnetic long-range order visible in the neutron experiments due to formation of alternating $\mathrm{Cu}-\mathrm{Cu}$ spin pairings along the $c$ direction. Unfortunately, this intellectually attractive picture has to be abandoned for the time being on the basis of the obtained structural information.

One may also wonder why a dimerization along the $a$ direction is not contained in our model and incidentally does not show up from the data. Such dimerization would become relevant close to the isotropic limit $J_{2} / J_{1} \approx 1,{ }^{20}$ and we are far below that according to the aforementioned estimates. Otherwise such dimerization is either an artifact caused by a finite number of chains transversally to $a$ or is even suppressed already relatively close to the isotropic case $\left(J_{2} / J_{1}=0.7\right){ }^{20}$ Although ref 20 offers a larger variety of order parameters, the most crucial features of the two kinds of spin liquids (a gapless one and another one with a small gap) are adequately reproduced even by our simpler model, which ultimately stems from refs 21 and 22 .

We also note that mechanostructural effects are known for other materials, for example, magnetostriction, which obviously is not the case because $\mathrm{CuNCN}$ is not a ferromagnet. Likewise, magnetostructural effects have been reported for materials with antiferromagnetic exchange. Such is the case for $\mathrm{CrF}_{2}$ and $\mathrm{CuF}_{2}$ when antiferromagnetic order sets in below the Néel temperature. ${ }^{37} \mathrm{CuNCN}$, however, is not an antiferromagnet but a spin liquid at any temperature. ${ }^{18}$ Another example is given by $\mathrm{SrCu}_{2}\left(\mathrm{BO}_{3}\right)_{2}$, to be described by a Heisenberg model with two exchange parameters. ${ }^{38}$ The topology of its exchange interactions, however, fundamentally differs from that of $\mathrm{CuNCN}$, and the temperature dependence of the susceptibility of $\mathrm{SrCu}_{2}\left(\mathrm{BO}_{3}\right)_{2}$ manifests the typical Curie-Weiss dependence of an antiferromagnet, ${ }^{39}$ unlike $\mathrm{CuNCN}$.

In conclusion, we have observed an anomalous temperature dependence of the $a$ lattice parameter in the nitrogencontaining analogue of cupric oxide, CuNCN, by means of high-resolution synchrotron powder diffraction. The effect manifests below $100 \mathrm{~K}$ and relates to the activated behavior of the ESR and SQUID measured spin susceptibilities. We have come up with an adequate, yet approximate, picture of the $2 \mathrm{D}$ resonating valence bond state proposed recently, and we were able to explain the observed structural features by this theory. Namely, a rearrangement of only a small fraction of all of the electrons induces a slight widening of the nearest $\mathrm{Cu}-\mathrm{Cu}$ distance. One finds a semiquantitative agreement between the experimental features and the theoretical model. Provided the theoretical interpretation of the observed effect is correct, our structural measurements provide clear evidence of hightemperature RVB states in CuNCN: first, the $1 \mathrm{D}-\mathrm{RVB}$ state as existing at room-temperature and responsible for the temperature-independent paramagnetism in the temperature region as low as $80-100 \mathrm{~K}$ and, second, the $2 \mathrm{D}$-RVB state below that temperature, responsible for the activation-like behavior of the magnetic susceptibility, the total absence of magnetic scattering as well as for the structural change reported in the present work. Because of the scale of the exchange parameters achieved in $\mathrm{CuNCN}$, the characteristic temperatures for the appearance of both RVB states are higher than those in any other material reported so far. ${ }^{8-11}$

\section{EXPERIMENTAL METHODS}

All preparative steps were based on highest purity educts or on educts of analytical grade. A dark-green aqueous solution of $\mathrm{CuCl}_{2}$ dihydrate and $\mathrm{H}_{2} \mathrm{NCN}$ was prepared in a roundbottomed flask, and a solution of $\mathrm{Na}_{2} \mathrm{SO}_{3}$ was added upon stirring; the solution became light-green. Concentrated ammonia was used to attain $\mathrm{pH}>9$, whereupon pale-white $\mathrm{Cu}_{4}(\mathrm{NCN})_{2} \mathrm{NH}_{3}$ precipitated. ${ }^{40}$ Upon stirring the suspension at room temperature overnight, the white precipitate was slowly oxidized by atmospheric oxygen to yield black and highly crystalline CuNCN. It was separated by filtration, washed with water, and dried in vacuo. ${ }^{14}$

High-resolution synchrotron powder diffraction data were collected at the Swiss Norwegian BeamLine (SNBL BM01B) at ESRF. ${ }^{41}$ The wavelength was calibrated with a $\mathrm{Si}$ standard NIST 640 c to 0.50195 and $0.50350 \AA$, respectively. The diffractometer is equipped with six counting channels, delivering six complete patterns collected simultaneously with 
a small $1.1^{\circ}$ offset in $2 \theta$. A Si(111) analyzer crystal is mounted in front of each $\mathrm{NaI}$ scintillator/photomultiplier detector. Powderous CuNCN was placed in a glass capillary of $\varnothing=0.9$ $\mathrm{mm}$. For low temperatures, the sample was placed in an exchange gas liquid He flow cryostat. In a first run $(\lambda=0.50195$ $\AA$ ), high-quality (in terms of statistics) data were collected for ca. $1 \mathrm{~h}$ at 293 and $5 \mathrm{~K}$ between 3.5 and $38.613^{\circ}$ ( $5 \mathrm{~K}: 35.496^{\circ}$ ) in $2 \theta$ with steps of $0.0025^{\circ}$ and $500 \mathrm{~ms}$ integration time per data point. During cooling between 293 and $90 \mathrm{~K}, 15$ rapid 5 min data acquisitions were taken between 5 and $13^{\circ}$ in $2 \theta$, also with steps of $0.0025^{\circ}$ but $100 \mathrm{~ms}$ integration time. During each scan, the temperature was continuously dropping, and the difference between the beginning and the end of the scan was never recorded to be larger than $5 \mathrm{~K}$. After the static $5 \mathrm{~K}$ measurement, additional static data were recorded while going up at $20,40,60$, and $80 \mathrm{~K}$. Here the temperature was stabilized to below $\pm 0.5 \mathrm{~K}$ to improve the accuracy. Data from all detectors were averaged to a step of $0.003^{\circ}$ with local software. Because an unusual trend of the lattice parameter $a$ was obtained from these data, in a second run $(\lambda=0.50350 \AA$, 16 bunch mode at $90 \mathrm{~mA}$, glass capillary of $\varnothing=1.0 \mathrm{~mm})$, data sets were recorded with the following temperature sequence: 120 , $110,100,90,70,50,30,10$, and $140 \mathrm{~K}$. The temperature was stabilized before each scan; the temperature stability can be estimated to be $\pm 0.2 \mathrm{~K}$. Continuous patterns were recorded between 3.5 and $37.8^{\circ}$ in $2 \theta$ with steps of $0.0025^{\circ}$ and $300 \mathrm{~ms}$ integration time per data point amounting to $\sim 1 \mathrm{~h}$ per scan.

The refinements of the diffraction patterns were performed using GSAS. ${ }^{42}$ The starting atomic coordinates ${ }^{14}$ were refined in space group $\mathrm{Cmcm}$ (no. 63). For all patterns, the background profile was fitted with a linear interpolation function with six coefficients. For the patterns at 293 and $5 \mathrm{~K}$, the profile of the diffraction peaks was modeled using a pseudo-Voigt function with one Gaussian and one Lorentzian coefficient and two parameters for the peak asymmetry $(S / L, H / L)$. Because the fwhm of the peaks showed a marked anisotropy, the $L_{i j}$ terms of the expression for the empirical microstrain anisotropy implemented in GSAS were tentatively refined. Three terms were needed to model the $5 \mathrm{~K}$ data and six were needed to model the $293 \mathrm{~K}$ data. All together, 26 variables were used to refine the $293 \mathrm{~K}$ data and 23 variables were used for the $5 \mathrm{~K}$ data. The refined pattern of the $293 \mathrm{~K}$ data is shown in the Supporting Information. Details of the refinements are summarized in Table 1 while selected interatomic distances are given in Table 2 . In terms of crystallographic resolution, data were refined down to $0.9 \AA$. To reduce the number of variables for the rapid measurements between 293 and $5 \mathrm{~K}$, no profile and atomic coordinates were refined. This reduced the number of variables to 12 . Instead, the parameters obtained for the $293 \mathrm{~K}$ data were used for all of these refinements. Additionally, one common thermal parameter $U_{\text {iso }}$ was refined. From these refinements, an unusual trend of the lattice parameter $a$ was obtained; that is, with decreasing temperature, $a$ increased. To confirm this finding in a second run (see above) patterns with a larger $2 \theta$ range and improved temperature stability were recorded. These data were also refined using GSAS with the following sets of parameters: six coefficients for the background profile (linear interpolation function), zero, lattice parameters $a, b$ and $c$, one common thermal parameter $U_{\text {iso, }}$ three atomic coordinates, scale, a pseudo-Voigt function with one Gaussian and one Lorentzian coefficient, and six $L_{\mathrm{ij}}$ terms for the empirical microstrain anisotropy. It was found that the peak asymmetry of the first
Table 1. Details of the Structural Investigations (SNBL/ ESRF) and Selected Crystallographic Parameters of CuNCN $(\mathrm{Cmcm}, \mathrm{Z}=4)$ at $293 \mathrm{~K}(\lambda=0.50195 \AA)$ and $100 \mathrm{~K}(\lambda=$ $0.50350 \AA$ )

\begin{tabular}{lll}
\multicolumn{1}{c}{$T(\mathrm{~K})$} & \multicolumn{1}{c}{293} & \multicolumn{1}{c}{100} \\
$a(\AA)$ & $2.99035(5)$ & $2.98815(3)$ \\
$b(\AA)$ & $6.1869(1)$ & $6.14842(7)$ \\
$c(\AA)$ & $9.4103(1)$ & $9.40080(9)$ \\
$V\left(\AA^{3}\right)$ & $174.101(8)$ & $172.715(4)$ \\
$w R_{\mathrm{P}}$ & 0.1496 & 0.1234 \\
$R_{\mathrm{P}}$ & 0.1141 & 0.0913 \\
$R_{\text {Bragg }}$ & 0.0615 & 0.0352 \\
no. of reflections & 132 & 117 \\
no. of refined parameters & 26 & 23 \\
refined angular range & $4^{\circ}<2 \theta<38.6^{\circ}$ & $9^{\circ}<2 \theta<37.5^{\circ}$ \\
$\mathrm{Cu}$ on $4 a$ & & \\
$\mathrm{~N}$ on $8 f$ & & $0.3804(3)$ \\
$y$ & $0.3823(4)$ & $0.3807(2)$ \\
$z$ & $0.3824(3)$ & \\
$\mathrm{C}$ on $4 c$ & & $0.3866(6)$ \\
$y$ & $0.3888(7)$ & \\
\hline
\end{tabular}

Table 2. Selected Interatomic Distances (Å) and Angles (deg) in CuNCN at 293 and $100 \mathrm{~K}$

$\begin{array}{ccc}T(\mathrm{~K}) & 293 & 100 \\ \mathrm{Cu}-\mathrm{N} & 1.998(2) 4 \times & 2.0078(14) 4 \times \\ \mathrm{C}-\mathrm{N} & 1.247(3) 2 \times & 1.229(2) 2 \times \\ \mathrm{N}-\mathrm{C}-\mathrm{N} & 176.3(5) & 176.5(4)\end{array}$

strong (002) reflection could not be modeled convincingly with the functions implemented in GSAS, so it was omitted in the final refinements. As an example, the refinement obtained from the $100 \mathrm{~K}$ data is also shown in the Supporting Information. Details of the refinements are summarized in Table 1, whereas selected interatomic distances are given in Table 2 . A negative thermal expansion of the lattice parameter $a$ was also obtained from these data, so the trend of the refinement results of the first data sets was fully confirmed. In Figures 2 and S2 in the Supporting Information, the results of both data sets are combined such that for high temperatures the results of the first run $(\lambda=0.50195 \AA)$ are shown in black, whereas the results of the second run $(\lambda=0.50350 \AA)$ are given in red. The results of the first run at low temperatures are not shown, but they are in agreement with the results of the second run showing a larger statistical spread. In addition, the effect seen is perfectly reproduced from preliminary neutron-diffraction data collected at the POWGEN instrument (SNS facility, Oak Ridge, TN).

\section{ASSOCIATED CONTENT}

\section{Supporting Information}

Rietveld refinement plots, course of the unit-cell volume as a function of the temperature, and sketch of the model derivation using refs 43 and 44 . This material is available free of charge via the Internet at http://pubs.acs.org.

\section{AUTHOR INFORMATION}

\section{Corresponding Author}

*E-mail: drons@HAL9000.ac.rwth-aachen.de.

Notes

The authors declare no competing financial interest. 


\section{ACKNOWLEDGMENTS}

This work was funded by Deutsche Forschungsgemeinschaft and partially supported by RFBR through the grant no. 10-0300155 dispatched to ALT. Dr. Andrej Zorko of Jožef Stefan Institute (Ljubljana, Slovenia) is acknowledged for sending numerical data on the $T$ dependence of $\boldsymbol{\eta}$ as extracted from the ESR measurements. ${ }^{18}$ We acknowledge valuable discussions with Dr. Evgeni Plekhanov (Università di Salerno, Italy), Dr. Serguei Zagoulaev (St. Petersburg University, Russia), Dr. Dmitry Chernyshov (SNBL, Grenoble, France), and Prof. Stefan Wessel (RWTH Aachen, Germany). We would also like to thank Volker L. Deringer for help with the artwork.

\section{REFERENCES}

(1) Pauling, L. The Dynamic Model of the Chemical Bond and its Application to the Structure of Benzene. J. Am. Chem. Soc. 1926, 48, 1132-1143.

(2) Pauling, L. A Resonating-Valence-Bond Theory of Metals and Intermetallic Compounds. Proc. R. Soc. London, Ser. A 1949, 196, 343362.

(3) Shaik, S.; Hiberty, P. C. A Chemist's Guide to Valence-Bond Theory; John Wiley \& Sons: Hoboken, NJ, 2008.

(4) Anderson, P. W. Resonating Valence Bonds: A New Kind of Insulator? Mater. Res. Bull. 1973, 8, 153-160.

(5) Anderson, P. W. The Resonating Valence Bond State in $\mathrm{La}_{2} \mathrm{CuO}_{4}$ and Superconductivity. Science 1987, 235, 1196-1198.

(6) Bednorz, J. G.; Müller, K. A. Possible High $T_{\mathrm{c}}$ Superconductivity in the $\mathrm{Ba}-\mathrm{La}-\mathrm{Cu}-\mathrm{O}$ System. Z. Phys. B 1986, 64, 189-193.

(7) Balents, L. Spin Liquids in Frustrated Magnets. Nature 2010, 464, 199-208.

(8) Shimizu, Y.; Miyagawa, K.; Kanoda, K.; Maesato, M.; Saito, G. Spin Liquid State in an Organic Mott Insulator with a Triangular Lattice. Phys. Rev. Lett. 2003, 91, 107001-1-107001-4.

(9) Coldea, R.; Tennant, D. A.; Tsvelik, A. M.; Tylczynski, Z. Experimental Realization of a 2D Fractional Quantum Spin Liquid. Phys. Rev. Lett. 2001, 86, 1335-1338.

(10) Coldea, R.; Tennant, D. A.; Tylczynski, Z. Extended Scattering Continua Characteristic of Spin Fractionalization in the TwoDimensional Frustrated Quantum Magnet $\mathrm{Cs}_{2} \mathrm{CuCl}_{4}$ Observed by Neutron Scattering. Phys. Rev. B 2003, 68, 134424-1-134424-6.

(11) Ono, T.; Tanaka, H.; Aruga Katori, H.; Ishikawa, F.; Mitamura, H.; Goto, T. Magnetization Plateau in the Frustrated Quantum Spin System $\mathrm{Cs}_{2} \mathrm{CuBr}_{4}$. Phys. Rev. B 2003, 67, 104431-1-104431-7.

(12) Meng, Z. Y.; Lang, T. C.; Wessel, S.; Assaad, F. F.; Muramatsu, A. Quantum Spin Liquid Emerging in Two-Dimensional Correlated Dirac Fermions. Nature 2010, 464, 847-852.

(13) Koo, H.-J.; Whangbo, M.-H. Magnetic Superstructures of Cupric Oxide $\mathrm{CuO}$ as Ordered Arrangements of One-Dimensional Antiferromagnetic Chains. Inorg. Chem. 2003, 42, 1187-1192.

(14) Liu, X.; Wankeu, M. A.; Lueken, H.; Dronskowski, R. A Novel Method for Synthesizing Crystalline Copper Carbodiimide, CuNCN. Structure Determination by X-ray Rietveld Refinement. Z. Naturforsch., B 2005, 60, 593-596.

(15) Liu, X.; Dronskowski, R.; Kremer, R. K.; Ahrens, M.; Lee, C.; Whangbo, M.-H. Characterization of the Magnetic and Structural Properties of Copper Carbodiimide, CuNCN, by Neutron Diffraction and First-Principles Evaluations of Its Spin Exchange Interactions. J. Phys. Chem. C 2008, 112, 11013-11017.

(16) Xiang, H.; Liu, X.; Dronskowski, R. Theoretical Reinvestigation of the Electronic Structure of CuNCN: the Influence of Packing on the Magnetic Properties. J. Phys. Chem. C 2009, 113, 18891-18896.

(17) Tsirlin, A.; Rosner, H. Uniform Spin-Chain Physics Arising from $\mathrm{N}-\mathrm{C}-\mathrm{N}$ Bridges in CuNCN, the Nitride Analog of the Copper Oxides. Phys. Rev. B 2010, 81, 024424-1-024424-10.

(18) Zorko, A.; Jeglič, P.; Potočnik, A.; Arčon, D.; Balčytis, A.; Jagličič, Z.; Liu, X.; Tchougréeff, A. L.; Dronskowski, R. Unconven- tional Magnetism in a Nitrogen-Containing Analog of Cupric Oxide. Phys. Rev. Lett. 2011, 107, 047208-1-047208-4.

(19) Tchougréeff, A. L.; Dronskowski, R. On the Properties of the Longitudinal RVB State in the Anisotropic Triangular Lattice. MeanField RVB Analytical Results. arXiv:1008.0182v2.

(20) Yunoki, S.; Sorella, S. Two Spin Liquid Phases in the Spatially Anisotropic Triangular Heisenberg Model. Phys. Rev. B 2006, 74, 014408-1-014408-31.

(21) Hayashi, Y.; Ogata, M. Possibility of Gapless Spin Liquid State by One-Dimensionalization. J. Phys. Soc. Jpn. 2007, 76, 053705-1053705-4.

(22) Hayashi, Y.; Ogata., M. Finite Temperature RVB Mean-Field Analysis of the Anisotropic Triangular Lattice Spin System. J. Phys. Conf. Ser. 2009, 150, 042053-1-042053-4.

(23) Read, N.; Sachdev, S. Valence-Bond and Spin-Peierls Ground States of Low-Dimensional Quantum Antiferromagnets. Phys. Rev. Lett. 1989, 62, 1694-1697.

(24) Capriotti, L.; Becca, F.; Parola, A.; Sorella, S. Resonating Valence Bond Wave Functions for Strongly Frustrated Spin Systems. Phys. Rev. Lett. 2001, 87, 097201-1-097201-4.

(25) Heidarian, D.; Sorella, S.; Becca, F. Spin-1/2 Heisenberg Model on the Anisotropic Triangular Lattice: From Magnetism to a OneDimensional Spin Liquid. Phys. Rev. B 2009, 80, 012404-1-012404-4.

(26) Lee, S.-S.; Lee, P. A. U(1) Gauge Theory of the Hubbard Model: Spin Liquid States and Possible Application to $\kappa$-(BEDT$\mathrm{TTF})_{2} \mathrm{Cu}_{2}(\mathrm{CN})_{3}$. Phys. Rev. Lett. 2005, 95, 036403-1-036403-4.

(27) Baskaran, G.; Zou, Z.; Anderson, P. W. The Resonating Valence Bond State and High- $T_{\mathrm{c}}$ Superconductivity - A Mean Field Theory. Solid State Commun. 1987, 63, 973-976.

(28) Tchougréeff, A. L.; Dronskowski, R. Tentative Structural Features of a Gapped RVB State in the Anisotropic Triangular Lattice. arXiv:1111.7210.

(29) Misurkin, I. A.; Ovchinnikov, A. A. Calculation of Bond Lengths and Valence Angles in Molecules with Conjugated Bonds. J. Struct. Chem. 1964, 5, 888-891 [in Russian].

(30) Misurkin, I. A.; Ovchinnikov, A. A. Calculation of the Vibration Frequencies of Molecules by the Molecular Orbital Method Using the Benzene Molecule as a Model. Opt. Spectr. 1964, 16, 228-233 [in Russian].

(31) Misurkin, I. A.; Ovchinnikov, A. A. Electronic Structure and Properties of Polymeric Molecules with Conjugated Bonds. Usp. Khim. 1977, 46, 1835-1870 [in Russian].

(32) Kivelson, S. A.; Rokhsar, D. S.; Sethna, J. P. Topology of the Resonating Valence-Bond State: Solitons and High- $T_{c}$ Superconductivity. Phys. Rev. B 1987, 35, 8865-8868.

(33) Coulson, C.; Golebievski, A. On Perturbation Calculations for the $\pi$-Electrons and their Application to Bond Length Reconsiderations in Aromatic Hydrocarbons. Proc. Phys. Soc. 1961, 78, 13101320.

(34) Peierls, R. E. Quantum Theory of Solids; Clarendon Press: Oxford, 1955.

(35) Belinskii, A. E.; Tchougréeff, A. L.; Misurkin, I. A. On the Different Types of the States of One-Dimensional System of Electrons in the Unrestricted Hartree-Fock Approximation. Teor. Eksp. Khim. 1989, 25, 513-520 [in Russian]; Theor. Exp. Chem. 1989, 25, 475481 [in English].

(36) Fukutome, H.; Sasai, M. Theory of Electronic Structures and Lattice Distortions in Polyacetylene and Inherent Peierls Systems, I. UHF Trends for Multielectron Method Adapted to the Long-range Coulomb Interaction and UHF States in Regular Bond Alternated Lattice. Prog. Theor. Phys. 1982, 67, 41-67.

(37) Chatterji, T.; Hansen, T. C. Magnetoelastic Effects in JahnTeller Distorted $\mathrm{CrF}_{2}$ and $\mathrm{CuF}_{2}$ Studied by Neutron Powder Diffraction. J. Phys.: Condens. Matter 2011, 23, 276007-1-276007-9.

(38) Vecchini, C.; Adamopoulos, O.; Chapon, L. C.; Lappas, A.; Kageyama, H.; Ueda, Y.; Zorko, A. Structural Distortions in the SpinGap Regime of the Quantum Antiferromagnet $\mathrm{SrCu}_{2}\left(\mathrm{BO}_{3}\right)_{2}$. J. Solid State Chem. 2009, 182, 3275-3281. 
(39) Kageyama, H.; Onizuka, K.; Yamauchi, T.; Ueda, Y.; Hane, S.; Mitamura, H.; Goto, T.; Yoshimura, K.; Kosuge, K. Anomalous Magnetizations in Single Crystalline $\mathrm{SrCu}_{2}\left(\mathrm{BO}_{3}\right)_{2}$. J. Phys. Soc. Jpn. 1999, 68, 1821-1823.

(40) Liu, X.; Müller, P.; Dronskowski, R. Synthesis and Crystal Structure of Ammine Copper(I) Cyanamide, $\mathrm{Cu}_{4}(\mathrm{NCN})_{2} \mathrm{NH}_{3}$. Z Anorg. Allg. Chem. 2005, 631, 1071-1074.

(41) Van Beek, W.; Safonova, O. V.; Wiker, G.; Emerich, H. SNBL, a Dedicated Beamline for Combined in situ X-ray Diffraction, X-ray Absorption and Raman Scattering Experiments. Phase Transitions 2011, 84, 726-732.

(42) Larson, A. C.; Von Dreele, R. B. General Structure Analysis System (GSAS); Los Alamos National Laboratory Report LAUR 86748; Los Alamos National Laboratory: Los Alamos, 2004.

(43) Ogata, M.; Fukuyama, H. The $t-J$ Model for the Oxide High- $T_{\mathrm{c}}$ Superconductors. Rep. Prog. Phys. 2008, 71, 036501-1-036501-45.

(44) Jahnke, E.; Emde, F.; Lösch, F. Tafeln Höhere Funktionen. 6. Auflage, neubearbeitet von F. Lösch; B. G. Teubner Verlagsgesellschaft: Stuttgart, Germany, 1960 [in German]. 